\title{
Norm Asymptotics of Orthogonal Polynomials for General Measures
}

\author{
M. F. Wyneken
}

\begin{abstract}
Let $\mu$ be a fixed positive unit Borel measure with infinite support in the unit disk. A carrier of $\mu$ is any Borel subset $B$ of the support for which $\mu(B)=1$, and another such measure $\nu$ is carrier-related to $\mu$ when it has the same carriers as $\mu$. Let $p_{n}(z, \nu)$ be the monic orthogonal polynomial of degree $n$ for $\nu$. We describe the possible asymptotics for the sequences $\left\{\left(\int\left|p_{n}(z, \nu)\right|^{2} d \nu\right)^{1 / 2 n}\right\}_{n \geq 1}$ which are associated to the set of measures carrierrelated to $\mu$.
\end{abstract}

\section{Introduction}

Let $\mu$ be a positive unit Borel measure with infinite support in the unit disk $\Delta=\{z:|z| \leq 1\}$. Let $P_{n}(z, \mu)$ denote the monic orthogonal polynomial of degree $n$ associated with $\mu$ for $n=0,1,2, \ldots$. Then let the norm of $\mu, N_{n}(\mu)$ be defined by the relationship

$$
\left(\int P_{m}(z, \mu) \overline{P_{n}(z, \mu)} d \mu\right)^{1 / 2}=N_{n}(\mu) \delta_{m, n}, \quad \delta_{m, n}= \begin{cases}1 & \text { if } m=n \\ 0 & \text { otherwise }\end{cases}
$$

In 1940 Erdös and Turán [3] generalized a fact about the norms of Jacobi polynomials by showing that if $\mu$ is supported on the interval $[-1,1]$, and if the absolutely continuous part of $\mu$ has a positive almost everywhere RadonNikodym derivative with respect to Lebesgue linear measure, then

$$
\lim _{n \rightarrow \infty}\left(N_{n}(\mu)\right)^{1 / n}=\frac{1}{2}
$$

However, if this derivative is not positive almost everywhere, the asymptotic behavior of the sequence $\left\{\left(N_{n}(\mu)\right)^{1 / n}\right\}_{n \geq 1}$ is seen to be dependent upon the capacities of the carriers, i.e., the Borel subsets of the support having unit measure. The relevance of potential theory was suggested by Erdös [3], and was developed by Ullman [6]-[9].

Date received: August 13, 1986. Date revised: March 2, 1987. Communicated by Paul Nevai AMS classification: 31A15, 33A65, 28A12, 41 A17.

Key words and phrases: Orthogonal polynomials for general measures, Norm, Carrier, Capacity. 
It is known that the limit point set of $\left\{\left(N_{n}(\mu)\right)^{1 / n}\right\}_{n \geq 1}$ is necessarily some closed interval subset of a certain maximal interval determined by the carriers. In this paper we make crucial use of a new and fundamental potential theoretic result of Ancona in conjunction with a technical lemma of Ullman to prove a theorem that will indicate in consequence that no further information about this limit point set can be obtained from measurements made on the carriers alone. The extension from measures having bounded linear support to those having bounded complex support is made possible by an application of a space-filling curve.

\section{Definitions and Statement of the Theorem}

Let $\mu$ be a positive unit Borel measure on $\Delta$. The support of $\mu$, denoted $S(\mu)$, is the smallest closed set having unit $\mu$-measure, and a Borel subset of the support having unit $\mu$-measure is called a carrier. When $S(\mu)$ is an infinite set, such a measure $\mu$ is called a weight measure.

The capacity of a bounded Borel set $B$ is taken as its inner logarithmic capacity, i.e., $C(B)=\sup _{K \subset B} C(K)$, where $K$ runs through all compact subsets of $B$, and $C(K)$ is the capacity of $K$ as derived from the logarithmic potential function $U(z, \mu)=\int \log \left(|z-t|^{-1}\right) d \mu(t)$ [5]. The following are properties of capacity [6]: for Borel sets $B_{1}$ and $B_{2}$,

(i) $B_{1} \subset B_{2} \Rightarrow C\left(B_{1}\right) \leq C\left(B_{2}\right)$,

(ii) $C\left(B_{1}\right)=0 \Rightarrow C\left(B_{2} \cup B_{1}\right)=C\left(B_{2}-B_{1}\right)=C\left(B_{2}\right)$,

(iii) If $K_{n}$ is compact, and $K_{n} \subset K_{n+1} \subset \Delta(n=1,2, \ldots)$, then

$$
\lim _{n \rightarrow \infty} C\left(K_{n}\right)=C\left(\bigcup_{n \geq 1} K_{n}\right)
$$

Throughout this paper, $\mu$ will denote a given weight measure with support in $\Delta$. A second weight measure $\nu$ is carrier-related to $\mu$ if it has the same carriers as $\mu$. Associated with the set of measures carrier-related to $\mu$ are two numbers, $C=\inf C(B)$, where the infimum is taken over all carriers, and $\bar{C}=C(S(\mu))$. We say that $\mu$ is a determined measure when $C=\bar{C}$, and that $\mu$ is undetermined otherwise. To see the existence of an undetermined measure let $\left\{r_{n}\right\}_{n \geq 1}$ be a dense subset of $\Delta$ and define a weight measure $\rho$ by $\rho\left(\left\{r_{n}\right\}\right)=2^{-n}, n=1,2, \ldots$ Then $S(\rho)=\Delta$ so $[C, \bar{C}]=[0,1]$.

Let $\nu$ be carrier-related to $\mu$, and let $\left[\left(N_{n}(\nu)\right)^{1 / n}\right]$ denote the limit point set of the sequence $\left\{\left(N_{n}(\nu)\right)^{1 / n}\right\}_{n \geq 1}$. It is known that $\left[\left(N_{n}(\nu)\right)^{1 / n}\right]$ is a closed interval subset of $[C, \bar{C}][6],[7],[9]$.

We now state the theorem which we will prove in the next section.

Theorem. Given a weight measure $\mu$ with support in $\Delta$, and given any closed interval subset $[\alpha, \beta]$ of $[C, \bar{C}]$, there exists a measure $\nu$ which is carrier-related to $\mu$ such that $\left[\left(N_{n}(\nu)\right)^{1 / n}\right]=[\alpha, \beta]$. 


\section{Proof of the Theorem}

The property of being carrier-related is equivalent to that of being mutually absolutely continuous [7], and hence it will suffice to construct a positive almost everywhere $(\mu)$ Borel measurable function $\omega(z)$ so that $d \nu=\omega(z) d \mu$ has the requisite property.

We will state four lemmas. Lemma 1 is due to Ancona, Lemma 2 is due to Ullman, and Lemma 3 is due to Szegö and Tonelli. Proofs of Lemmas 2 and 4 appear in the last section.

Let $K$ be a compact subset of $C$ having positive capacity and let $\Omega$ be the unbounded component of the complement of $K$ in $C$. Let $\mu_{K}$ denote the equilibrium measure for $K\left[5\right.$, p. 55]. The equilibrium potential $U\left(z, \mu_{K}\right)$ is harmonic for $z \in \Omega$, and when it is continuous on $C$ we say that $K$ is regular.

Lemma 1 [1], [2]. Let $B$ be a Borel subset of $\mathbf{C}$ with $C(B)>0$, and let $\varepsilon>0$. Then $B$ has a regular compact subset $\mathscr{K}$ with $C(\mathscr{K})>C(B)-\varepsilon$.

Lemma 2 [7]. Let $\mu$ be a weight measure with support $S(\mu)$ in $\Delta$. Let $B$ be $a$ Borel subset of $S(\mu)$, and let $\mathscr{K}$ be a regular compact subset of $S(\mu)$. Assume that $\mu\left(B \cap N_{\varepsilon}(z)\right)>0$ for each $z \in \mathscr{K}$ and all $\varepsilon>0$. Let $n$ be a positive integer. Then:

(i) There is a nonnegative Borel measurable function $\tau_{n}(z)$, called a transfer function, with the properties that $\int \tau_{n}(z) d \mu=n^{-2}$ and $A_{n}=\left\{z: \tau_{n}(z)>0\right\}$ is a compact subset of $B$,

(ii) There is a sequence of positive integers $\left\{a_{n}\right\}_{n \geq 1}$ with the property that $\lim _{n \rightarrow \infty}\left(a_{n}\right)^{1 / n}=1$ and such that if $P_{n}(z)$ is any polynomial of degree $n$, then

$$
\int\left|P_{n}(z)\right|^{2} \tau_{n}(z) d \mu \geq\left(a_{n}\right)^{-1}\left(\left\|P_{n}(z)\right\|_{\mathscr{H}}\right)^{2},
$$

where $\left\|P_{n}(z)\right\|_{\mathscr{K}}=\max _{z \in \mathscr{K}}\left|P_{n}(z)\right|$.

Lemma $3\left[5\right.$, p. 73]. Let $K$ be a compact subset of $\Delta$, and let $M_{n}(K)=\inf \left\|P_{n}(z)\right\|_{K}$, where the infimum is taken over all monic polynomials $P_{n}(z)$ of degree $n$.

(i) If $K$ contains at least $n$ points, there is a unique monic Chebychev polynomial $T_{n}(z, K)$ of degree $n$ satisfying $\left\|T_{n}(z, K)\right\|_{K}=M_{n}(K)$.

(ii) If $K$ is infinite, then $\lim _{n \rightarrow \infty}\left(M_{n}(K)\right)^{1 / n}=C(K)$.

(iii) For $z \in \Delta,\left|T_{n}(z, K)\right| \leq 2^{n}$.

Lemma 4 [10]. For each $\gamma \in[\underline{C}, \bar{C}]$ there exists an $F_{\sigma}$ carrier $B_{\gamma}=\bigcup_{p \supseteq 1} K_{\gamma, p}$ such that (i) $C\left(B_{\gamma}\right)=\gamma$ and (ii) $\mu\left(K_{\gamma, p} \cap N_{\varepsilon}(z)\right)>0$ for each $z \in K_{\gamma, p}$ and all $\varepsilon>0$, where $N_{\varepsilon}(z)=\{w:|w-z|<\varepsilon\}$. Moreover, we may assume $\mu\left(K_{\gamma, p}\right)>0$, and that $\alpha \leq \beta$ implies $K_{\alpha, p} \subset K_{\beta, p}(p=1,2, \ldots)$.

We will now prove the theorem. Assume first that $\alpha$ is positive. Let $B_{\alpha}=$ $\bigcup_{p \geq 1} K_{\alpha, p}$ and $B_{\beta}=\bigcup_{p \geq 1} K_{\beta, p}$ be $F_{\sigma}$ carriers as described in Lemma 4 having capacities $\alpha$ and $\beta$, respectively, with $\mu\left(K_{\alpha, p}\right)>0$ and $K_{\alpha, p} \subset K_{\beta, p}(p=1,2, \ldots)$. 
Let $\left\{\varepsilon_{p}\right\}_{p \geq 0}$ be a sequence of positive numbers decreasing to zero, and let $\gamma$ represent both of the numbers $\alpha$ and $\beta$. For each integer $p$ let $\mathscr{K}_{\gamma, p}$ be a regular compact subset of $K_{\gamma, p}$ satisfying $C\left(\mathscr{K}_{\gamma, p}\right)>C\left(K_{\gamma, p}\right)-\varepsilon_{p}$. From the Wiener criterion [5, p. 104], we may assume that $\mathscr{K}_{\gamma, p} \subset \mathscr{K}_{\gamma, p+1}$. By Lemma 2, there exists for each integer $n$, transfer functions $\tau_{\gamma, p, n}(z)$ supported on compact subsets $A_{\gamma, p, n}$ of $K_{\gamma, p}$, such that for any polynomial $P_{n}(z)$ of degree $n$,

$$
\int\left|P_{n}(z)\right|^{2} \tau_{\gamma, p, n}(z) d \mu \geq\left(a_{\gamma, p, n}\right)^{-1}\left(\left\|P_{n}(z)\right\|_{\varkappa_{\gamma, p}}\right)^{2},
$$

with $\lim _{n \rightarrow \infty}\left(a_{\gamma, p, n}\right)^{1 / n}=1$.

Since $B_{\alpha}$ is a carrier we may assume $A_{\beta, p, n} \subset B_{\alpha}$. Let $a_{\gamma, p}$ be an integer such that $n \geq a_{\gamma, p}$ implies that $1 \leq\left(a_{\gamma, p, n}\right)^{1 / n} \leq 1+\varepsilon_{p}$, and let $a_{p}$ be an integer greater than $\max \left\{a_{\alpha, p}, a_{\beta, p}, a_{p-1}\right\}\left(p=1,2, \ldots ; a_{0}=0\right)$. The construction is as follows. For a compact set $K$ with $C(K) \leq \gamma$, let $[K, \gamma, \varepsilon]$ denote the least positive integer for which $n \geq[K, \gamma, \varepsilon]$ implies $\left\|T_{n}(z, K)\right\|_{K} \leq(\gamma+\varepsilon)^{n}$ (Lemma 3). Let $[\Sigma, \varepsilon]$ denote the least positive integer $m$ for which $\sum_{n \geq m} n^{-2} \leq \varepsilon$. Now let $x_{0}=x_{1}=0$, and, for $p=1,2, \ldots$, let

$$
f_{2 p-1}=\max \left\{a_{p}, x_{2 p-2}+1,\left[\Sigma,(\alpha / 2)^{2 x_{2 p-1}}\right]\right\}
$$

and

$$
f_{2 p}=\max \left\{x_{2 p-1}+1,\left[K_{\alpha, p} \cup \bigcup_{1 \leq k \leq p} A_{\beta, k, x_{2 k}}, \alpha, \varepsilon_{p}\right],\left[K_{\beta, p+1}, \beta, \varepsilon_{p}\right]\right\} .
$$

By the principle of recursive definition [4, p. 10], there is a unique sequence $\left\{x_{j}\right\}_{j \geq 0}$ which satisfies $x_{2 p}=f_{2 p-1}$ and $x_{2 p+1}=f_{2 p}(p=1,2, \ldots)$. Let $x_{2 p}=t_{p}, x_{2 p+1}=$ $s_{p}(p=0,1, \ldots)$, and $L_{p}=K_{\alpha, p} \cup \bigcup_{1 \leq k \leq p} A_{\beta, k, x_{2 k}}(p=1,2, \ldots)$. Then with this change of notation,

$$
\begin{array}{r}
\sum_{n \geqslant t_{p}} n^{-2} \leq(\alpha / 2)^{2 s_{p-1},} \\
\left\|T_{s_{p}}\left(z, L_{p}\right)\right\|_{L_{p}} \leq\left(\alpha+\varepsilon_{p}\right)^{s_{p}},
\end{array}
$$

and

$$
\left\|T_{n}\left(z, K_{\beta, p+1}\right)\right\|_{K_{\beta, n+1}} \leq\left(\beta+\varepsilon_{p}\right)^{n} \quad \text { for } \quad n \geq s_{p}
$$

Now let

$$
\sigma_{p}(z)=\chi_{K_{\alpha, p}}(z)\left(\mu\left(K_{\alpha, p}\right)\right)^{-1}\left((\alpha / 2)^{2 s_{p-1}}-(\alpha / 2)^{2 s_{r}}\right),
$$

where $\chi_{\kappa_{\alpha, p}}(z)$ is the characteristic function of the set $K_{\alpha, p}$, and let

$$
\omega(z)=c \sum_{q \geq 1}\left(\sigma_{q}(z)+\tau_{\beta, q, r_{q}}(z)+\sum_{t_{4}<n \leqslant t_{q+1}} \tau_{\alpha, q, n}(z)\right),
$$

where $c$ is chosen so that $\int \omega(z) d \mu=1$. 
We must demonstrate four inequalities:

(i) $\lim \sup _{n \rightarrow \infty}\left(N_{n}(\nu)\right)^{1 / n} \leq \beta$,

(ii) $\lim \inf _{n \rightarrow \infty}\left(N_{n}(\nu)\right)^{1 / n} \geq \alpha$,

(iii) $\lim \sup _{p \rightarrow \infty}\left(N_{s_{p}}(\nu)\right)^{1 / s_{p}} \leq \alpha$,

(iv) $\lim \inf _{p \rightarrow \infty}\left(N_{t_{p}}(\nu)\right)^{1 / t_{r}} \geq \beta$.

In demonstrating inequalities (ii) and (iv) we use the fact that $\left\|P_{n}(z)\right\|_{K} \geq(C(K))^{n}$ for monic polynomials $P_{n}(z)$ of degree $n$ and compact sets $K$ [5, p. 62].

(i) For $s_{p}<n \leq s_{p+1}$,

$$
\begin{aligned}
\left(N_{n}(\nu)\right)^{2}= & \int\left|P_{n}(z, \nu)\right|^{2} d \nu \\
= & \int\left|P_{n}(z, \nu)\right|^{2} \omega(z) d \mu \\
\leq & \int\left|T_{n}\left(z, K_{\beta, p+1}\right)\right|^{2} \omega(z) d \mu \\
= & \int_{K_{\beta, p+1}}\left|T_{n}\left(z, K_{\beta, p+1}\right)\right|^{2} \omega(z) d \mu+\int_{S(\mu)-K_{\beta, p+1}}\left|T_{n}\left(z, K_{\beta, p+1}\right)\right|^{2} \omega(z) d \mu \\
\leq & \left(\beta+\varepsilon_{p}\right)^{2 n} \\
& +c 2^{2 n} \int_{S(\mu)-K_{\beta, p+1}} \sum_{q \geq 1}\left(\sigma_{q}(z)+\tau_{\beta, q, t_{q}}(z)+\sum_{t_{q}<n \leq t_{q+1}} \tau_{\alpha, q, n}(z)\right) d \mu \\
\leq & \left(\beta+\varepsilon_{p}\right)^{2 n}+c 2^{2 n}\left((\alpha / 2)^{2 s_{p+1}}+\sum_{q \geq p+2}\left(t_{q}\right)^{-2}+\sum_{n>t_{p+2}} n^{-2}\right) \\
\leq & \left(\beta+\varepsilon_{p}\right)^{2 n}+c 2^{2 n}\left((\alpha / 2)^{2 s_{p+1}+}+(\alpha / 2)^{2 s_{p+1}+}+(\alpha / 2)^{\left.2 s_{p+1}\right)}\right. \\
\leq & (1+3 c)\left(\beta+\varepsilon_{p}\right)^{2 n} .
\end{aligned}
$$

(ii) For $t_{p}<n \leq t_{p+1}$,

$$
\begin{aligned}
\left(N_{n}(\nu)\right)^{2} & =\int\left|P_{n}(z, \nu)\right|^{2} d \nu \\
& =\int\left|P_{n}(z, \nu)\right|^{2} \omega(z) d \mu \\
& \geq c \int_{A_{\alpha, p, n}}\left|P_{n}(z, \nu)\right|^{2} \tau_{\alpha, p, n}(z) d \mu \\
& \geq c\left(a_{\alpha, p, n}\right)^{-1}\left(\left\|P_{n}(z, \nu)\right\|_{\mathcal{K}_{\alpha, p}}\right)^{2} \\
& \geq c\left(1+\varepsilon_{p}\right)^{-n}\left(C\left(K_{\alpha, p}\right)-\varepsilon_{p}\right)^{2 n}
\end{aligned}
$$


(iii) $\left(N_{s_{p}}(\nu)\right)^{2}=\int\left|P_{s_{p}}(z, \nu)\right|^{2} d \nu$

$$
\begin{aligned}
& =\int\left|P_{s_{p}}(z, \nu)\right|^{2} \omega(z) d \mu \\
& \leq \int\left|T_{s_{p}}\left(z, L_{p}\right)\right|^{2} \omega(z) d \mu \\
& =\int_{L_{p}}\left|T_{s_{p}}\left(z, L_{p}\right)\right|^{2} \omega(z) d \mu+\int_{S(\mu)-L_{p}}\left|T_{s_{p}}\left(z, L_{p}\right)\right|^{2} \omega(z) d \mu \\
& \leq\left(\alpha+\varepsilon_{p}\right)^{2 s_{p}} \\
& +c 2^{2 s_{p}} \int_{S(\mu)-L_{p}} \sum_{q \geq 1}\left(\sigma_{q}(z)+\tau_{\beta, q, t_{q}}(z)+\sum_{\iota_{4}<n \leq t_{q+1}} \tau_{\alpha, q, n}(z)\right) d \mu \\
& \leq\left(\alpha+\varepsilon_{p}\right)^{2 s_{p}}+c 2^{2 s_{p}}\left((\alpha / 2)^{2 s_{p}}+\sum_{q \geqslant p+1}\left(t_{q}\right)^{-2}+\sum_{n>t_{, j+1}} n^{-2}\right) \\
& \leq\left(\alpha+\varepsilon_{p}\right)^{2 s_{p}}+c 2^{2 s_{r}}\left((\alpha / 2)^{2 s_{p}}+(\alpha / 2)^{2 s_{p}}+(\alpha / 2)^{2 s_{p}}\right) \\
& \leq(1+3 c)\left(\alpha+\varepsilon_{p}\right)^{2 s_{p}} \text {. }
\end{aligned}
$$

(iv)

$$
\begin{aligned}
\left(N_{t_{p}}(\nu)\right)^{2} & =\int\left|P_{t_{p}}(z, \nu)\right|^{2} d \nu \\
& =\int\left|P_{t_{p}}(z, \nu)\right|^{2} \omega(z) d \mu \\
& \geq c \int_{A_{\beta, r_{p} t_{p}}}\left|P_{t_{p}}(z, \nu)\right|^{2} \tau_{\beta, p, t_{p}}(z) d \mu \\
& \geq c\left(a_{\beta, p, t_{p}}\right)^{-1}\left(\left\|P_{n}(z, \nu)\right\|_{\mathcal{K}_{\beta, p}}\right)^{2} \\
& \geq c\left(1+\varepsilon_{p}\right)^{-t_{p}}\left(C\left(K_{\beta, p}\right)-\varepsilon_{p}\right)^{2 t_{p}}
\end{aligned}
$$

The remaining cases when $\alpha$ is zero are handled similarly. There is no need for transfer functions for a carrier of zero capacity; however, infinite sets are required for the use of Chebychev polynomials. Suppose $B_{0}$ is a carrier of zero capacity. Since a carrier $B_{0}$ is an infinite set, and we may take $B_{0}=\bigcup_{p \geq 1} K_{0, p}$ where $K_{0 . p}$ is compact, $\mu\left(K_{0, p}\right)>0$, and $K_{0, p} \subset K_{0, p+1}(p=1,2, \ldots)$, we may then assume the existence of a convergent sequence $\lim _{p \rightarrow x} z_{p}=z_{1}$ where $z_{p} \in$ $K_{0, p}-K_{0, p-1}(p=2,3, \ldots)$. Since $C\left(\left\{z_{1}\right\}\right)=0$, assume $z_{1} \in B_{0}$ so $\left\{z_{p}\right\}_{p \geq 1}$ is an infinite compact subset of $B_{0}$. Hence we may assume further that $K_{0, p}$ itself is an infinite set $(p=1,2, \ldots)$.

If $0=\alpha<\beta$, for $p=1,2, \ldots$, let

$$
\begin{gathered}
t_{p}=\max \left\{a_{p}, t_{p-1}+1,\left[\Sigma,\left(\varepsilon_{p-1} / 2\right)^{2 s_{p-1}}\right]\right\}, \\
s_{p}=\max \left\{s_{p-1}+1,\left[K_{0, p} \cup \bigcup_{1 \leq k \leq p} A_{\beta, k, t_{k}}, 0, \varepsilon_{p}\right],\left[K_{\beta, p+1}, \beta, \varepsilon_{p}\right]\right\}, \\
\sigma_{p}(z)=\chi_{K_{0, p}}(z)\left(\mu\left(K_{0, p}\right)\right)^{-1}\left(\left(\varepsilon_{p-1} / 2\right)^{2 s_{p-1}}-\left(\varepsilon_{p} / 2\right)^{2 s_{n}}\right),
\end{gathered}
$$


and let

$$
\omega(z)=c \sum_{p \geq 1}\left(\sigma_{p}(z)+\tau_{\beta, p, t_{p}}(z)\right) .
$$

If $0=\alpha=\beta$, for $p=1,2, \ldots$, let

$$
\begin{gathered}
s_{p}=\max \left\{s_{p-1}+1,\left[K_{0, p}, 0, \varepsilon_{p}\right]\right\}, \\
\sigma_{p}(z)=\chi_{\kappa_{0, p}}(z)\left(\mu\left(K_{0, p}\right)\right)^{-1}\left(\left(\varepsilon_{p-1} / 2\right)^{2 s_{p}}-\left(\varepsilon_{p} / 2\right)^{2 s_{p+1}}\right),
\end{gathered}
$$

and

$$
\omega(z)=c \sum_{p \geq 1} \sigma_{p}(z)
$$

\section{Proof of the Lemmas}

Proof of Lemma 2. For $z \in \mathrm{C}$, let $G(z)=\log \left(C(\mathscr{K})^{-1}\right)-U\left(z, \mu_{\mathscr{K}}\right)$, and let $G_{\delta}=$ $\max _{z \in \mathscr{H}_{\delta}}|G(z)|$ where $\mathscr{K}_{\delta}=\left\{z \in \mathbf{C}: \min _{w \in \mathscr{H}}|z-w| \leq \delta\right\}$. It has been shown [7] that if $z^{*} \in \mathscr{K}$ is chosen such that $\left|p_{n}\left(z^{*}\right)\right|=\left\|p_{n}(z)\right\|_{\mathcal{K}}$ and we set $d_{n}=$ [ $\left.n \exp \left(n G_{2 / n}\right)\right]+1$ (brackets [ ] indicating the least integer function), then, for any $z$ such that $\left|z-z^{*}\right| \leq\left(2 d_{n}\right)^{-1}$, it follows that $\left|p_{n}(z)\right| \geq\left(\frac{1}{2}\right)\left\|p_{n}(z)\right\|_{\mathscr{H}}$. Let $x_{i}=$ $-1+i\left(6 d_{n}\right)^{-1}, y_{j}=-1+j\left(6 d_{n}\right)^{-1}$, and let $S_{i, j}=\left\{(x, y) \in \mathrm{C}: x_{i-1} \leq x \leq x_{i}, y_{j-1} \leq y \leq\right.$ $\left.y_{j}\right\}$, so that $S(\mu) \subset \bigcup_{1 \leq i, j \leq 12 d_{n}} S_{i, j}$. Let $S_{n, k}, k=1,2, \ldots, m_{n}, m_{n} \leq\left(12 d_{n}\right)^{2}$, denote those squares $S_{i, j}$ which intersect $\mathscr{K}$, and let $z_{n, k} \in \mathscr{K} \cap S_{n, k}$. Let $A_{n, k}$ be a compact subset of $B \cap N_{\left(4 d_{n}\right)^{-1}}\left(z_{n, k}\right)$ with $\mu\left(A_{n, k}\right)>0$. Hence if $z^{*} \in S_{n, k^{*}}$ for some $k^{*}$, $1 \leq k^{*} \leq m_{n}$, then, for any $w \in A_{n, k^{*}},\left|z^{*}-w\right| \leq\left|z^{*}-z_{n, k^{*}}\right|+\left|z_{n, k^{*}}-w\right| \leq\left(2 d_{n}\right)^{-1}$.

Now let $A_{n}=\bigcup_{1 \leq k \leq m_{n}} A_{n, k}$, let

$$
\tau_{n}(z)=\left(n^{2} m_{n}\right)^{-1} \sum_{1 \leq k \leq m_{n}} \chi_{A_{n, k}}(z)\left(\mu\left(A_{n, k}\right)\right)^{-1},
$$

and let $a_{n}=4 n^{2} m_{n}$. The fact that $\lim _{n \rightarrow \infty} a_{n}^{1 / n}=1$ follows from the fact that $\mathscr{K}$ is regular.

Proof of Lemma 4. For $B \subset S(\mu)$, let $Z(B)=\left\{z \in B: \mu\left(B \cap N_{\delta}(z)\right)=0\right.$ for some positive $\delta\}$ and let $P(B)=B-Z(B)$. Observe that $\mu(Z(B))=0$; for if $z \in Z(B)$, then $\mu\left(B \cap N_{\delta}(z)\right)=0$ for some $\delta>0$, and there is a rational point $w$ and a positive rational $\eta$ such that $z \in N_{\eta}(w) \subset N_{\delta}(z)$, and hence $Z(B) \subset \bigcup_{\eta, w} B \cap$ $N_{\eta}(w)$, a countable union of sets of measure zero.

Next observe that $P(B)$ is closed if $B$ is closed. To see this, let $\left\{z_{n}\right\}_{n \geq 1}$ be a sequence in $P(B)$ converging to $z$ in $B$. If $z$ is not in $P(B)$, then there exists a positive $\delta$ such that $\mu\left(B \cap N_{\delta}(z)\right)=0$. Then, for sufficiently small positive $\varepsilon$ and sufficiently large $n, 0<\mu\left(B \cap N_{\varepsilon}\left(z_{n}\right)\right) \leq\left(B \cap N_{\delta}(z)\right)$, which is a contradiction.

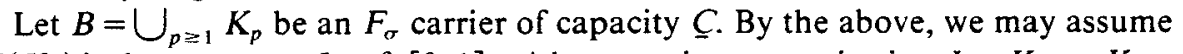
$Z\left(K_{p}\right)$ is the empty set. Let $f:[0,1] \rightarrow \Delta$ be a continuous surjection. Let $K_{p, x}=K_{p} \cup$ $\{f(t): 0 \leq t \leq x\} \cap S(\mu)$, let $I_{p, x, y}=K_{p, y}-K_{p, x}$, and let $C(x)=C\left(\bigcup_{p \geq 1} P\left(K_{p, x}\right)\right)$. It remains to show that $C(x)$ is a continuous nondecreasing function on $[0,1]$ whose range is $[\underline{C}, \bar{C}]$. 
Observe that $C(0)=C\left(\bigcup_{p \geq 1} P\left(K_{p, 0}\right)\right)=C\left(\bigcup_{p \geq 1} K_{p}\right)=C$, and $C(1)=$ $C\left(\bigcup_{p \geq 1} P\left(K_{p, 1}\right)\right)=C(S(\mu))=\bar{C}$. Next, observe that $P\left(B_{1}\right) \subset P\left(B_{2}\right)$ if $B_{1} \subset B_{2}$. For if $z \in P\left(B_{1}\right)$, then there exists a positive $\delta$ such that $\mu\left(B_{2} \cap N_{\delta}(z)\right)>$ $\mu\left(B_{1} \cap N_{\delta}(z)\right)>0$, and hence $z \in P\left(B_{2}\right)$. It then follows that $C(x)$ is nondecreasing, since $C(x)=C\left(\bigcup_{p \geq 1} P\left(K_{p, x}\right)\right) \leq C\left(\bigcup_{p \geq 1} P\left(K_{p, y}\right)\right)=C(y)$ if $x<y$.

Let $\varepsilon>0$ and $B \subset S(\mu)$. Then there exists $\delta>0$ such that for each $A \subset S(\mu)$ with $C(A)<\delta$ we have $C(B)-\varepsilon \leq C(B-A)$ and $C(B \cup A) \leq C(B)+\varepsilon$. This follows from two facts [5, pp. 56,63]: (i) If $B_{1} \cup B_{2}=B_{3} \subset\left\{z:|z| \leq \frac{1}{2}\right\}$, then $\left(\log C\left(B_{3}\right)\right)^{-1} \geq\left(\log C\left(B_{1}\right)\right)^{-1}+\left(\log C\left(B_{2}\right)\right)^{-1}$. (ii) If $a \in C$, then $C(\{a z: z \in B\})=$ $|a| C(B)$.

For $x<y$ we have

$$
\begin{aligned}
Z\left(K_{p, x}\right)-Z\left(K_{p, y}\right)= & \left\{z \in K_{p, x}: \mu\left(K_{p, x} \cap N_{\delta}(z)\right)=0 \text { for some positive } \delta\right\} \\
& -\left\{z \in K_{p, y}: \mu\left(K_{p, y} \cap N_{\delta}(z)\right)=0 \text { for some positive } \delta\right\} \\
= & \left\{z \in K_{p, x}: \mu\left(K_{p, x} \cap N_{\delta}(z)\right)=0 \text { and } \mu\left(K_{p, y} \cap N_{\delta}(z)\right)\right. \\
& >0 \text { for all sufficiently small positive } \delta\} \\
\subset & \left\{z \in K_{p, x}: \mu\left(I_{p, x, y} \cap N_{\delta}(z)\right)>0 \text { for all positive } \delta\right\} \\
\subset & \left\{z \in K_{p, x}: \mu\left(\{f(t): x \leq t \leq y\} \cap N_{\delta}(z)\right)>0 \text { for all positive } \delta\right\} \\
\subset & \{f(t): x \leq t \leq y\}, \text { since this last set is closed. }
\end{aligned}
$$

Let $D_{\delta}(x)=[x-\delta, x+\delta] \cap[0,1]$. We then have

$$
\begin{aligned}
C(x+\delta) & =C\left(\bigcup_{p \geq 1} K_{p, x+\delta}-Z\left(K_{p, x+\delta}\right)\right) \\
& =C\left(\bigcup_{p \geq 1}\left(K_{p, x}-Z\left(K_{p, x}\right)\right) \cup\left(I_{p, x, x+\delta}-Z\left(K_{p, x+\delta}\right)\right) \cup\left(Z\left(K_{p, x}\right)-Z\left(K_{p, x+\delta}\right)\right)\right) \\
& \leq C\left(\bigcup_{p \geq 1} P\left(K_{p, x}\right) \cup f\left(D_{\delta}(x)\right),\right.
\end{aligned}
$$

and

$$
\begin{aligned}
C(x-\delta) & =C\left(\bigcup_{p \geq 1} K_{p, x-\delta}-Z\left(K_{p, x-\delta}\right)\right) \\
& =C\left(\bigcup_{p \geq 1}\left(K_{p, x}-Z\left(K_{p, x}\right)\right)-I_{p, x-\delta, x}-\left(Z\left(K_{p, x-\delta}\right)-Z\left(K_{p, x}\right)\right)\right) \\
& \geq C\left(\bigcup_{p \geq 1} P\left(K_{p, x}\right)-f\left(D_{\delta}(x)\right)\right) .
\end{aligned}
$$

By continuity, $\lim _{\delta \rightarrow 0} C\left(f\left(D_{\delta}(x)\right)\right)=0$. Hence, for given $\varepsilon>0$, there exists $\delta>0$ such that $C(x)-\varepsilon \leq C(x-\delta) \leq C(x+\delta) \leq C(x)+\varepsilon$, so $C(x)$ is continuous.

Acknowiedgment. The author wishes gratefully to acknowledge his thesis adviser, Professor Joseph L. Ullman. 


\section{References}

1. A. Ancona (1983): Demonstration d'une conjecture sur la capacité et effilement. C.R. Acad. Sci. Paris, Sér. 1, 297:393-395.

2. A. ANCONA (1984): Sur une conjecture concernant la capacité et l'efflement. In: Theorie du Potential, Orsay 1983. Lecture Notes in Mathematics, vol. 1096. New York: Springer-Verlag, pp. 10-21.

3. P. ERdöS, P. TURÁN (1940): On interpolation, III. Ann. of Math., 41:510-553.

4. H. L. ROYDEN (1968): Real Analysis, 2nd edn. New York: Macmillan.

5. M. Tsus (1959): Potential Theory in Modern Function Theory. Tokyo: Maruzen.

6. J. L. UlLman (1972): On the regular behavior of orthogonal polynomials. Proc. London Math. Soc., 24:119-148.

7. J. L. Ullman (1985): Orthogonal polynomials for general measures, II. In: Polynômes Orthogonaux et Applications, Proceedings, Bar-le-Duc, 1984. Lecture Notes in Mathematics, vol. 1171. New York: Springer-Verlag, pp. 247-254.

8. J. L. ULLMAN, M. F. WYNEKEN (1986): Weak limits of zeros of orthogonal polynomials. Constr Approx. 2:339-347.

9. J. L. Ullman, M. F. WyNEkEN, L. Ziegler (1986): Norm oscillatory weight measures J. Approx. Theory, 46:204-212.

10. M. F. WYNEKEN (1985): Norm behavior of orthogonal polynomials. Thesis, University of Michigan.

M. F. Wyneken

Department of Mathematics

University of Michigan-Flint

Flint

Michigan 48502-2186

U.S.A. 